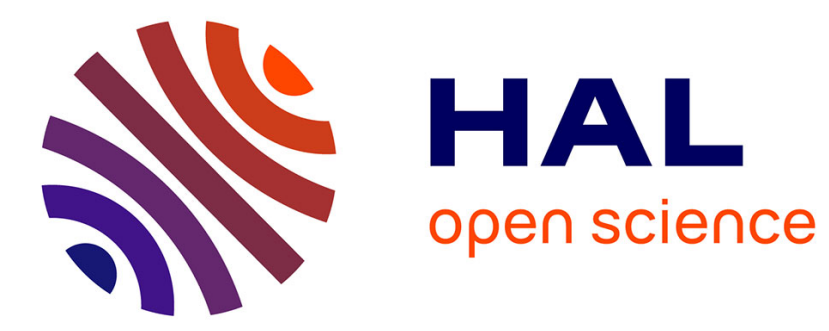

\title{
La décroissance, une pensée antimoderne ?
}

Fabrice Flipo

\section{To cite this version:}

Fabrice Flipo. La décroissance, une pensée antimoderne ?. Chimères : revue des schizoanalyses, 2012, 76, pp.69-79. 10.3917/chime.076.0069 . hal-00958093

\section{HAL Id: hal-00958093 https://hal.science/hal-00958093}

Submitted on 11 Mar 2014

HAL is a multi-disciplinary open access archive for the deposit and dissemination of scientific research documents, whether they are published or not. The documents may come from teaching and research institutions in France or abroad, or from public or private research centers.
L'archive ouverte pluridisciplinaire HAL, est destinée au dépôt et à la diffusion de documents scientifiques de niveau recherche, publiés ou non, émanant des établissements d'enseignement et de recherche français ou étrangers, des laboratoires publics ou privés. 


\section{La décroissance, une pensée antimoderne?}

\section{A propos de l'écologisme aujourd'hui}

Fabrice Flipo, philosophe, maitre de conférences à ETOS / TEM Research

Auteur de Justice, nature et liberté (Parangon, 2007), Le développement durable (Bréal, 2007), La décroissance, 10 questions pour comprendre et en débattre (La Découverte, 2010).

Membre du Comité de Rédaction de la revue Mouvements.

Co-organisateur du premier colloque international sur la décroissance, Paris, 2008.

\section{Introduction}

Le constat a souvent été fait que l'écologisme peut être entendu comme mouvement porteur d'une " éthique environnementale », cherchant à " protéger la nature ». Cette revendication a fréquemment été critiquée, au motif qu'elle est vide en ce qui concerne les relations humaines, et qu'elle ouvre la porte à une critique de la modernité, c'est-à-dire de l'universalisme. Dominer la nature, c'est une conquête moderne, les époques antérieures ont au contraire été le lieu de la superstition. Sans cela on ne peut pas expliquer le progrès technique que la modernité a généré. D'où diverses tentatives venant du libéralisme ou du marxisme qui ont voulu proposer des alternatives jetant les bases d'une « écologie sociale » qui permette d'éviter les éléments antimodernes décelées chez les écologistes. L'écologie est une affaire trop sérieuse pour être laissée aux écologistes, en quelque sorte, elle devait revenir aux spécialistes du social et de l'universalisme que sont les libéraux ou les socialistes. Ces critiques sont renouvelées à l'endroit de la décroissance.

Nous montrons ici que cette lecture omet tout un pan de la critique écologiste, qui met en cause la société technicienne - une critique éminemment « sociale », compte-tenu de l'importance de la technique dans l'organisation des sociétés modernes. D'Illich à Ellul, nombreux sont les penseurs dont le travail n'est pas sans rappeler les efforts « d'organisation » de la société par les premiers réformistes, consternés par la misère humaine provoquée par les débuts de l'industrialisation. Et ce n'est pas un élément marginal des revendications des associations puisqu'aujourd'hui plus aucune n'ignore cette dimension.

Cette pensée de la technique présente de plus une dimension qui cherche à renouer avec l'universalisme, en cherchant à démontrer que tel ou tel choix technique est le seul qui soit généralisable au monde entier. Or à l'inverse le « social » classiquement défendu par les partisans du « développement », socialiste ou libéral, ignore cette dimension, et se condamne à l'abstraction. Pour en sortir, c'est notamment l'accumulation qui devrait être mise en cause - indépendamment de toute thèse sur la propriété. Nous montrons que ce défaut d'universalisme a d'ailleurs été bien été perçu dans les pays qui ont été rangés en bloc, souvent contraints et forcés, dans la catégorie des "sous-développés ». Ce que la critique de la société technicienne pointe de manière indirecte est la question de la civilisation : qui aura le leadership, au $21 \mathrm{e}$ siècle, dans ce domaine?

\section{La question du social dans l'écologisme}

La question écologique a souvent été abordée, dans les travaux de philosophie, par l'angle de l'éthique environnementale. La revue internationale de référence s'appelle d'ailleurs " Environmental Ethics ». De cette réflexion sur la nature est née une insatisfaction : si on prend en compte la nature, que deviennent les relations humaines ? Beaucoup ont vu la pensée écologiste comme n'apportant pas de réponse à cette question, ou apportant de mauvaises réponses, insensibles aux acquis universalistes de la modernité.

Dans le cadre de ce colloque, impossible de ne pas citer le petit livre de Félix Guattari, Les trois écologies. Guattari affirmait que l'écologie devait cesser d'être liée à l'image d'une minorité d'amoureux de la nature et d'experts, il suggérait, en plus de l'écologie " environnementale », de développer l'écologie " sociale », notre mode d'être avec les autres, et l'écologie « mentale », le rapport à notre propre psyché. De là une « écologie généralisée » (p47). La question écosophique, disait-il, est trop importante pour être abandonnée à certains courants « archaïsants et folklorisants » (p47) voire même autoritaires. On ne saurait être plus clair.

Cette critique est reprise à l'endroit de la décroissance. Du côté libéral, la décroissance est scandaleuse à 
plus d'un titre. Elle s'autorise à parler des besoins, un domaine qui a été réservé à la sphère privée et résolu, dans ses grandes lignes, par le marché. Dès lors les partisans de la décroissant ne seraient-ils pas un totalitarisme rampant ? Ses partisans ne voudrait-ils pas imposer des rationnements, des modes de vie obligatoires, abolir les classes pour restaurer les statuts (Stände), figer la mobilité sociale ? A ce titre, la décroissance n'est-elle pas une idée réactionnaire ? Et puis cette idée n'est-elle pas indécente, tout simplement : alors que tant de gens souffrent de la faim dans le monde, et qui par conséquent ne demandent qu'à voir leur travail employé, comment peut-on leur dire de se serrer la ceinture ?

Du côté marxiste la proposition est tout aussi scandaleuse. La perspective d'une décroissance ne peut être qu'idéologique, c'est précisément le discours que tenaient les patrons aux ouvriers, quand les salaires ne dépassaient pas le niveau des subsistances, alors que dans le même temps des fortunes colossales s'élevaient sur le commerce et l'industrie. Un nom symbolise cela : Malthus, qui affirmait, en 1798, qu'un " homme qui nait dans un monde déjà occupé, si sa famille n'a pas les moyens de le nourrir, ou si les riches n'ont pas besoin de son travail, cet homme, dis-je, n'a pas le moindre droit à réclamer une portion quelconque de nourriture, et il est réellement de trop sur la terre. Au grand banquet de la nature, il n'y a point de couvert mis pour lui. La nature lui commande de s'en aller, et elle ne tardera pas à mettre elle-même cet ordre à exécution $»^{1}$. La décroissance ne peut être reçu que comme un discours idéologique justifiant le refus de partager. Protéger la nature, c'est protéger l'ordre établi, et justifier la misère. Avant la bourgeoisie, le clergé tenait déjà ce discours. Elle aussi enjoignait de protéger la Création, qui comprenait dans son ordre Providentiel des riches et des pauvres. Ici manifester une révérence à l'endroit de la nature ne peut être que suspecté de vouloir naturaliser un ordre social.

Ces deux critiques pointent dans le même sens que Guattari : l'écologisme possède un dangereux penchant antimoderne. Le fait de ne se soucier que de la nature conduit à négliger les acquis de la modernité. II est donc urgent de ne pas laisser l'écologie aux écologistes. La seule écologie qui soit acceptable doit rester dans les cadres de la modernité.

Qu'est-ce à dire ?

Côté libéral, Dominique Bourg, dans $L^{\prime}$ 'homme-artifice ${ }^{2}$, rappelle que la modernité est l'ère d'une relation rationnelle et scientifique à la nature, quand à l'époque d'Aristote l'art n'était vu que comme imitation de la nature et à l'époque moyenâgeuse comme devant respecter la Création divine. La modernité est l'ère de la démocratie, non pas la démocratie étouffante d'Athènes ou pire encore de Sparte mais la démocratie de marché, innovante et changeante, héritière de la « liberté des Modernes » définie par Benjamin Constant. Dans ce cadre le bon écologisme est facile à définir, Luc Ferry l'a fait dans un ouvrage a succès : c'est celui qui permet au marché de s'adapter aux nouvelles demandes, "vertes », des consommateurs.

Les sociologues de leur côté, citons notamment Anthony Giddens, ont caractérisé les mouvements écologistes par leur critique de la technique et plus largement de l'aménagement du territoire ${ }^{3}$. Alors que les courants d'inspiration marxiste laissaient ce sujet de côté, tout un courant de la sociologie en a déduit que les écologistes amenaient un supplément de "réflexivité », thème cher à Giddens, dans la modernité - en jouant le rôle de " watchdogs ", des contre-pouvoirs limitant les excès des marchés. Ainsi est né le courant dit de la "modernisation écologique », qui considère les associations écologistes comme des partenaires dans un contrat social renouvelé. Ainsi sont « internalisées » les « externalités ».

Et côté marxiste ? Jacques Bidet attire l'attention sur l'organisation et la relation discursive, à côté du marché ${ }^{4}$. II reprend largement le schéma de Bourg, mais met en évidence un décalage, entre la forme idéale (ou « métastructure »), la fiction sur fond de laquelle se déroulent les relations sociales, et la réalité, écart où se glisse l'injustice - le formel ne coïncide pas avec le réel. Le capitalisme, ici, de par la domination de la valeur qu'il met en place, détruit l'environnement, et ne peut se limiter lui-même. II faut donc le limiter, ici comme dans d'autres domaines, de l'extérieur, par une « étaticité » démocratique (Bidet, p. 305), fondée sur la valeur d'usage. La question écologique n'appelle donc pas d'analyse spécifique, l'abolition du capitalisme, au profit de l'organisation, est toujours à l'ordre du jour.

Dominique Bourg et Marx s'accordent en outre pour dire que l'enjeu philosophique de la relation à la nature

1 In Pierre Leroux, De la ploutocratie ou le gouvernement des riches (1842); Malthus et les économistes (1846). Consultables sur Gallica

2 Dominique Bourg, L'homme-artifice, Gallimard, 1996.

3 Anthony Giddens, Les conséquences de la modernité, L'Harmattan, 1994.

4 Jacques Bidet, Théorie générale, PUF, 1999. 
est la technique. L'un pense que la technique va trouver les solutions demandées par les consommateurs, l'autre que l'organisation palliera aux défauts du marché. Peut-on être si confiant ? Le diagnostic est-il le bon?

\section{Les contradictions de la modernisation écologique}

Face à ces critiques, l'écologisme et l'objection de croissance ont quelques arguments à faire valoir.

Les libéraux devraient d'abord remarquer que la validité de leurs théories reposent toutes sur une croissance illimitée, puisque la " catallaxie » théorisée par Hayek suppose des évolutions « Pareto-optimales » pour pouvoir se poursuivre. Le sentier suivi est donc précis : c'est celui des rendements croissants, du déplacement des fonctions de production vers le haut. Voir le marché comme un lieu qui se plie à volonté aux désirs des consommateurs, comme le suggère Luc Ferry, est une vue de l'esprit, car le déploiement technologique, responsable des $7 / 8$ e de la croissance selon le prix Nobel Robert Solow, est orienté dans un seul sens : l'accumulation. Or ce c'est ce sens-là qui a des conséquences néfastes sur la biodiversité, par exemple, puisque la mécanisation implique la standardisation, qui à son tour implique l'homogénéisation. C'est aussi la machine qui consomme les énergies fossiles. C'est pourtant d'elle que les libéraux attendent le salut. Ainsi Paul Samuelson et la profession des économistes se moque encore des " prophètes de malheur » qui prédisent des épuisements qui ne viennent jamais. Se moquer ne peut être une réponse sérieuse au défi de la substitution des ressources, d'autant que la donne a changé : plus personne ne nie la réalité des changements climatiques, de l'épuisement du pétrole. Personne ne pense que la voiture électrique sera un jour moins cher que la voiture à essence, une étude détaillée des possibles dans les différents secteurs de la production montrerait qu'il n'y a pas d'innovation de rupture qui permette d'entrevoir une « dématérialisation » de l'économie. S'il ne restait que la foi, l'espérance dans la technique, ce serait un curieux retournement de situation, les plus millénaristes ne seraient pas ceux qui sont montrés du doigt. Dans ce contexte, le processus malthusien va donc être activé : la croissance des riches ne pourra se faire que par décroissance des pauvres. L'ordre libéral ne sera plus intégrateur, il deviendra destructeur. " Internaliser » les " externalités » pose donc un problème bien plus profond que prévu, c'est la modernité qui est mise en cause.

La théorie marxiste avait bien vu le caractère univoque de cette accumulation. Le marché n'est pas une machine à agréger n'importe quels votes, comme semble le croire Luc Ferry. II ne peut pas produire de " croissance verte ». Et cela parce qu'il n'est pas seulement un mécanisme d'agrégation mais un mécanisme d'accumulation. Marx, à la suite de Sismondi, a bien montré comment le capital ne peut se reproduire qu'en s'élargissant. Cet élargissement annihile tous les efforts qui sont faits pour rendre les produits « verts ». Un produit vert ne peut être produit que s'il est moins cher qu'un autre, et s'il est moins cher alors des moyens sont dégagés pour consommer autre chose. De là ce fait que l'amélioration écologique des produits est toujours plus que compensée par leur multiplication, et que l'effet général sur l'écosystème reste nul. C'est « l'effet rebond ». "Le marché » pose donc un problème, tant qu'il est régi par l'accumulation. Or un marché sans accumulation reste à théoriser. $\mathrm{Ni}$ « l'organisation » ni la primauté de la " valeur d'usage » sur la « valeur d'échange », ni l'instauration de la prééminence du " politique » sur "l'économique » et « le profit », comme le suggèrent les auteurs marxistes, ne sont suffisant pour penser les conséquences d'un changement technique permettant de réduire de $80 \%$ les émissions de gaz à effet de serre. Le marxisme scientifique, de Staline à la doctrine officielle de l'Union Soviétique, n'a pas remis en cause l'évolution technique. L'histoire était vue comme évoluant principalement sur la base de la dialectique entre les forces productives et les rapports de production. II prévoyait la socialisation progressive des moyens de production comme solution, une thèse que reprennent aujourd'hui Negri \& Hardt, autour des technologies de l'information. Ces thèses, n'ayant pas pensé une autre technologie, ont maintenu un « rapport capitaliste » à la nature. Les régimes politiques qui se sont réclamés du socialisme et du communisme ont d'ailleurs continué d'accumuler, de capitaliser, bien que la propriété privée des moyens de production ait été abolie. Qu'a donc signifié « l'abolition de la valeur » qu'ils ont cru mettre en œuvre ? Ces questions sont largement restées en friche, jusqu'à ce jour. J.B. Foster, par exemple, se penchant sur l'écologisme de Marx, ne s'interroge pas réellement sur les fondements des choix technologiques ${ }^{5}$.

5 Voir John Bellamy Foster, Marx's ecology - materialism and nature, New York, Monthly Review Press, 2000; Ecology against capitalism, Monthly Review Press, 2002. Fabrice Flipo, L'écologie politique est-elle réactionnaire?, Sens Public, 16 juin 2010. http://www.sens-public.org/spip.php?article754 


\section{L'universalisme antimoderne de l'écologisme et des objecteurs de croissance}

Se contraindre à penser à l'intérieur de la modernité restreint considérablement les possibles. Et affirmer en bloc que la modernité incarne l'universalisme, c'est négliger un argument central des organisations écologistes et des objecteurs de croissance : celui qui affirme que notre mode de vie n'est pas universalisable.

Pour l'affirmer, le critère que proposent certaines associations est clair. II s'agit de " l'espace écologique ", lequel renvoie finalement à la célèbre « clause de Locke » qui affirme ceci : l'appropriation est permise pourvu « nul autre ne soit lésé par celui qui s'approprie une parcelle quelconque de terre en l'améliorant, car il en resterait assez, d'une qualité aussi bonne, et même plus que ne pourraient utiliser les individus qui n'étaient pas encore pourvus. Si bien qu'en réalité, le bornage que l'un effectuerait à son profit ne réduirait jamais la part des autres ${ }^{6}$. Cette clause peut bien sûr être étendue aux autres êtres vivants, c'est ici que se jouent une partie des débats relevant de « l'éthique environnementale », dans lesquels nous n'entrerons pas ici.

Un tel critère justifie d'opposer le droit d'existence voire le droit au développement (reconnu en 1984 par I'ONU), inaliénables, au droit d'occupation ou de commerce. Nul ne peut vivre sans échange métabolique avec le milieu naturel ; nul ne peut se vendre ou vendre son " corps inorganique », pour reprendre la formule de Marx, sans tomber en esclavage. C'est un tel critère qui justifie que le montant de la réduction de l'impact matériel des modes de vie s'élève à $80 \%$ - et pas à $50 \%$, comme ce serait le cas si les responsabilités étaient communes, mais pas différenciées. La différenciation des responsabilités traduit l'ampleur supérieure, par habitant, de l'impact cumulé des modes de vie des pays " développés " sur les biens communs planétaires. Ceci suffit en soi à remettre en cause ce " développement ». Et il justifie aussi le choix de techniques plus égalitaires, souvent fondées sur des circuits courts - ainsi des fameuses AMAP ${ }^{7}$, par exemple.

Or ces pratiques sont, au regard de tous les critères de modernité existants, profondément antimodernes, et même antidéveloppementiste. Toutes les théories du développement, jusqu'ici, d'Adam Smith à Douglass North, de Pierre Leroux à Gustav Schmoller, ont estimé que le développement c'est l'expansion de la division du travail, de l'organisation du travail, pas sa contraction.

Cet universalisme antimoderne ou partisan d'une autre modernité n'est pas impensable. Côté marxiste, Moishe Postone, dans un ouvrage dont les analyses ont été reprises à leur compte par nombre d'objecteurs de croissance ${ }^{8}$, explique que le marxisme traditionnel ne pouvait pas aboutir à un régime différent car sa théorie de la valeur se situe en réalité dans le capitalisme, et non hors du capitalisme. Le rapport entre le travail et le capital est un rapport de répartition, et non d'abolition de la valeur. C'était certes déjà excessivement important : la condition des ouvriers, dans la première partie du $19 \mathrm{e}$ siècle, était extraordinairement misérable, leurs conditions de vie étaient bien inférieures à celles que connaissent les sociétés paysannes. Les mouvements ouvriers ont largement été à l'origine de la domestication du capitalisme sauvage, ainsi que de divers mécanismes redistributifs. Contrairement à ce qu'espéraient les socialismes utopiques, férocement critiques à l'endroit du luxe et de la mode, qui menaient l'industrie de manière chaotique et capricieuse à cette époque-là, le marxisme scientifique d'une part et la consommation de masse de l'autre ont intégré les revendications ouvrières dans le capitalisme, aboutissant à des systèmes techniques similaires, à des « développements » étrangement analogues. Côté libéral, le soutien affiché par des partis politiques comme les Verts à l'économie sociale et solidaire conduit aussi à renouer avec certains éléments du socialisme et du réformisme, l'associationnisme, le mutuellisme etc.

En renvoyant « l'écologisme » à la simple « protection de la nature », en occultant la question de la technique, Guattari a considérablement appauvri sa lecture du mouvement. Et il y a plus : sa position, dans le fond, était " productiviste », selon le terme consacré. II mettait en effet de grands espoirs dans les machines numériques, se faisait prophétique et estimait qu'il viendra un temps où il serait nécessaire d'engager d'immenses programmes pour réguler les rapports entre oxygène, ozone et dioxyde de carbone

6 John Locke, Traité du gouvernement civil, p. 93.

7 Association pour le Maintien de l'Agriculture Paysanne

8 Moishe Postone, Temps, travail et domination sociale, Mille et Une Nuits, 2009, Ed. Orig. 1993. 
dans l'atmosphère. Cela n'a rien d'une nécessité : le vivant assure déjà cette régulation atmosphérique. Y voir un destin, c'est rester dans la logique de la machine, qui est celle de l'accumulation, de la valeur, produit et produira toujours plus d'exclus. Pour les objecteurs de croissance tels que Paul Ariès ou Serge Latouche, Guattari attribue à la fois trop de pouvoir aux désirs humains, et pas assez. Trop de pouvoir, au sens où il ignore les logiques du monde dans lequel nous baignons, notamment celle de la technologie, de l'accumulation. Et pas assez, au sens où il ne met pas en question notre destin technologique, il nous commande seulement de l'apprivoiser, d'en surmonter les excès - pas d'en infléchir la direction.

Pour quelle raison notre destin serait-il de déstabiliser la régulation atmosphérique, par exemple, pour faire appel aux machines cyclopéennes et utopiques que Guattari appelle de ses voeux ? Qu'est-ce qui nous y contraint ? C'est précisément cette question, qu'il ne discute pas, qui est posée par le courant critique de la technique. Bourg ou Foster passent eux aussi à côté de ce sujet. Telle n'est pas la perspective des mouvements écologistes qui entendent au contraire remettre en cause cette techno-logie, cette logique du développement technique, pour faire place à un autre possible, un autre destin. De manière similaire, le journal La Décroissance entend clairement se démarquer de la simple protection de la nature et poser d'emblée le problème en termes d'écologie mentale et sociale : c'est la société de consommation qui nous aliène, disent-ils. Et de revendiquer le programme du Conseil National de la Résistance de 1945.

Parmi les éléments qui remettent effectivement en cause la modernité, l'objection de croissance en exhibe donc un qui semblera peut-être inattendu : son manque d'universalité. Inattendu, car si les Modernes défendent la modernité, c'est au nom de son universalité.

Condamner l'écologisme au nom de son antimodernisme, au motif que la modernité est " évidemment » synonyme d'universalité, omet donc de saisir ce qui motive ces mouvements. Les branches qui sont apparemment «folkorisantes " sont peut-être celles qui ont le mieux perçu ce que pourrait être une " écologie sociale », " sociale » étant pris au sens que lui donnaient les premiers socialistes, c'est-à-dire animé du souci de ne laisser personne sur le bord de la route. A contrario la posture de Guattari ne se distingue pas clairement d'un écologisme "bobo ", de classe moyenne et supérieure, du fait de son manque de précision sur ce sujet-clé qu'est le social. Tout est renvoyé à une éthique, à une sagesse qui ne relèverait que d'une attitude individuelle.

Remettre en cause la modernité est-il dramatique, pour les non-modernes ? Est-ce un message qui est impossible à entendre, dans un monde épris de développement et assoiffé de « modernisation »?

Non, une étude approfondie de la pensée d'un pays tel que l'Inde montre qu'il existe depuis longtemps une critique autochtone de la modernité, au nom de l'universel. La modernité paraît en effet largement se confondre avec l'occidentalisation. Gandhi est peut-être la figure la plus connue de ce regard critique. Gandhi n'est pas dépassé. Le groupe postmarxiste des subaltern studies, notamment, a repris son héritage, dans les années 80. II a fait école dans le monde entier. Le Sommet des peuples sur la justice climatique, organisé à Cochabamba au Vénézuela, a été dominé par le mot d'ordre autochtone du " buen vivir », démontre aussi cela. L'intérêt souvent observé des écologistes pour les peuples classés " primitifs » pousse cette logique jusqu'à son terme. La période postcoloniale est propice à la déconstruction de ce qu'Edward Saïd a appelé « l'orientalisme ». Les non-Modernes s'avèrent ne pas être systématiquement irrationnels et engoncés dans des sociétés oppressives. Des recherches récentes portant sur l'ethnobotanique montrent que les superstitions des soi-disant sauvages ont souvent un sens écologique profond. A la lumière de l'écologie, le totémisme que Durkheim était contraint de ranger dans une obscure " religion » prend un tout autre sens. Et inversement : pour Ramachandra Guha, un auteur indien, il ne fait pas de doute que les Occidentaux, en cherchant à protéger l'éléphant, le tigre, s'accrochent à leurs totems - le problème est qu'ils le font au nom de la science, et qu'ils ne respectent pas les totems des autres ${ }^{9}$. Le fait est d'autant plus patent que les totems occidentaux sont plus volontiers des machines que des êtres vivants.

\section{Conclusion : sortir de l'ambiguïté}

Ceci nous permet d'embrayer sur notre conclusion. Nous avons tenté de montrer que les critiques de l'écologie politique ont raison de penser que ce courant politique contient des éléments antimodernes, se

9 Ramachandra Guha, "The authoritarian biologist and the arrogance of anti-humanism », The Ecologist, vol. $27, n^{\circ} 1$, January / february 1997, pp $14-20$. 
ramenant essentiellement à la « défense de la nature ». Guattari, comme beaucoup d'autres, ramène l'écologisme à la question de " l'éthique environnementale », et face à cela posent la question du social, c'est-à-dire la question des rapports humains. Ce n'est pas totalement faux, aussi l'interrogation est-elle partiellement pertinente : le mouvement écologiste s'est bel et bien en partie construit autour de naturalistes qui critiquaient la société industrielle, au nom d'un certain romantisme naturaliste. Paul Ehrlich, René Dubos, Rachel Carson, Barry Commoner etc. étaient tous biologistes dont les propos peuvent résonner comme malthusiens, au sens où ils laissent en partie dans le flou la question de la différenciation des responsabilités au sein du groupe des « humains».

Cette lecture est toutefois réductrice, pour deux raisons. Tout d'abord Guattari, mais aussi Bourg, Foster et avec eux un grand nombre d'autres critiques de l'écologisme, avancent des alternatives qui ne sont guère crédibles, et qui de plus échouent à faire la preuve matérielle de leur universalisme. Ensuite ramener l'écologisme à la protection de la nature c'est n'avoir vu connu qu'un seul aspect, très limité, du mouvement. Comme l'a montré Dominique Simonnet dans l'un des meilleurs livres écrits à ce jour sur le sujet ${ }^{10}$, ou encore Sylvie Ollitrault (« Militer pour la planète »), le mouvement écologiste est en grande partie animé d'une critique de la technique - ainsi toutes les grandes associations de " protection de la nature " se sontelles emparées de la question des choix technologiques - , voire même d'une aspiration émancipatrice et égalitaire - que traduit bien la référence à l'espace écologique.

L'écologisme a longtemps consisté à expliquer que la nature était notre " corps inorganique » et qu'on ne pouvait pas l'instrumentaliser à volonté sans que cela ne finisse par être nuisible. Il passait le plus gros de son énergie à montrer que la nature avait une " valeur intrinsèque ", qu'elle ne pouvait pas être considérée comme une simple ressource pour le système économique.

Aujourd'hui tout le monde est en passe d'être convaincu, le débat s'est déplacé sur les différentes manières d'être « vert ». L'écologisme est donc contraint d'évoluer et de se positionner sur des questions qui pouvaient jusque-là rester dans l'ambiguïté. Malthus est l'une de ces questions-clé. L'union sacrée (« ni droite, ni gauche ", pour résumer) ne pouvait être une stratégie payante que pour autant que la question de la différenciation des responsabilités ne se posait pas directement. Aujourd'hui, elle devient contreproductive, car c'est sur cette base que les publics à convaincre vont se décider. Le fond d'antimodernisme n'est pas près de se dissiper. L'universalisme n'a pas disparu pour autant. Nous pouvons donc bien « être progressistes sans devenir réactionnaires », comme le suggérait un livre de Jean-Paul Besset, l'un des conseillers de Nicolas Hulot. Mais nous pouvons aussi être d'authentiques réactionnaires, et soutenir un système qui n'a d'universel que l'égoïsme de ses promoteurs. 